\title{
PERFORMANCE ANALYSIS OF IS-95 CDMA CELLULAR SYSTEM USING NONCOHERENT $M$-ARY ORTHOGONAL MODULATION WITH OTHER CELL INTERFERENCE
}

\author{
K. Sivanesan, W. H. Lam \\ Dept. of Electrical \& Electronic Engg., The University of Hong Kong, \\ Pokfulam Road, Hong Kong
}

\begin{abstract}
The bit error rate (BER) performance of the $M$-ary DS/CDMA cellular system in the presence of an additive white Gaussian noise (AWGN) and interference from other cells has been investigated. The data frame structure of the IS-95 system was adopted. The system operated in an asynchronous mode with perfect power control and voice activity factor of $3 / 8$. Both the hard handoff and the soft handoff were considered respectively. A close form expression for the BER performance has been derived for the single cell CDMA system whereas numerical integration was employed for the multiple cells CDMA systems instead.
\end{abstract}

\section{INTRODUCTION}

Direct Sequence Code Division Multiple Access (DS/CDMA), has been widely studied [1-5,7] for digital cellular mobile radio communications. The study of the $M$-ary DS/CDMA in a single cell system employing $M$-ary orthogonal modulation and noncoherent demodulation in the presence of additive white Gaussian noise had been reported in literatures [1-3]. The multiple access interference was modeled as a Gaussian noise and perfect power control was assumed. Hong et al. [3] analyzed the effects of pilot PN sequence on the same system. Both the Gold and msequence were employed as the pilot sequences, respectively. For the application of digital cellular systems, Gilhousen et al. [4] further examined the DS/CDMA system having interference from other cells in a multiple-cell cellular system. The interference is assumed to be Gaussian distributed. Hard handoff was employed and the modulation technique was not considered. A close-form expression of outage probability was derived for the condition that the probability of $\left(\mathrm{BER}<10^{-3}\right)$ is equal to that of $\left(E_{b} / N_{0}>7 \mathrm{~dB}\right)$. The interference from other cells of the system employing different handoff algorithms was further investigated in literature [5].

In this paper, an analytical method has been derived to investigate the error performance of the multiple-cell
DS/CDMA communication systems in the presence of AWGN and interference from other cells. The system employed the $M$-ary Walsh orthogonal modulation and the coherent demodulation with non-coherent combining. The system is operated in an asynchronous mode and perfect power control is assumed. Although the IS-95 CDMA [8] data frame structure has been adopted, the analysis is applicable to a similar systems having higher chip rate transmission.

\section{SYSTEM MODEL}

The transmitter/receiver system model for the $M$-ary DS/CDMA reverse link over the AWGN channel is adopted as in [3]. It employs $M$-ary Walsh orthogonal code of length $M$ chips to represent $m$ data bits and each Walsh codes is of duration $T$. The Walsh code is further spread by the pilot $P N$ sequences $P_{I}^{i}(t)$ and $P_{\mathrm{Q}}^{i}(t)$ at the ratio of 1:4 for the in-phase and quadrature channel, respectively. The superscript $i$ denotes the $i$-th cell for the pilot code in a multiple-cell cellular system. The $M$-ary orthogonal signal $W^{0, k}(t)$ for the $k^{\text {th }}$ user in the $0^{\text {th }}$ cell can be written as

$W^{0, k}(t)=\sum_{v=-\infty}^{+\infty} W_{v}^{k}(t) P_{T}(t-v T)$

where $P_{\tau}(t)=1$ for $0 \leq t \leq \tau$; otherwise $P_{\tau}(t)=0$. In the above equation, the subscript $v$ of the symbol $W_{v}^{k}(t)$ denotes the $v^{t h}$ Walsh symbol in the code set $W_{p}(t)$ given by

$W_{p}(t)=\sum_{j=0}^{M-1} w_{p, j} P_{T_{W}}\left(t-j T_{W}\right) ; p=1,2, \ldots M$

where $w_{p, j}$ is the Walsh chip of duration $T_{w}$ (i.e., $M T_{n}$. $=T)$ and $w_{p, j} \in\{+1,-1\}$. The transmitted signal $s_{k}(t)$ of the $k^{\text {th }}$ user in the $0^{\text {th }}$ cell is thus written as

$s_{k}(t)=\sqrt{P} W^{0, k}\left(t-\tau_{k}\right) A^{0}\left(t-\tau_{k}\right) e^{j\left(\omega_{c} t+\theta_{k}\right)}$

where $\theta_{\kappa}, \tau_{\kappa}, \omega_{c}$ and $P$ represent the phase of $k^{\text {th }}$ carrier, the relative time delay, common carrier frequency and the common signal power, respectively. Each cell 
employs a signature sequence which consists of a pair of unique pilot PN sequences to distinguish the cell identity. For instance, the $i^{\text {th }}$ cell signature sequence is given by $A^{i}(t)$ where $A^{i}(t)=P_{I}^{i}(t)+j P_{Q}^{i}(t), \quad P_{I}^{i}(t)=$ $\sum_{j=-\infty}^{+\infty} p_{l, j}^{i} P_{T_{c}}\left(t-j T_{c}\right)$ and $p_{Q}^{i}(t)=\sum_{j=-\infty}^{+\infty} p_{Q, j}^{i} P_{T_{c}}\left(t-j T_{c}\right)$. The pilot PN sequences $P_{I}^{i}(t)$ and $P_{Q}^{i}(t)$ are of length $N$ for the inphase (I) and quadrature (Q) channels, respectively. Their amplitudes $p_{I, j}^{i}$ and $p_{Q, j}^{i}$ are of \pm 1 . The chip duration is of $T_{c}$, where $T_{w}=4 T_{c}$. For the simplicity of analysis, the half chip offset of the PN sequence in the quadrature branch is neglected. Consider an asynchronous cellular system that consists of $(n+1)$ cells. Define $K$ as the number of active users in the $0^{\text {th }}$ cell and $L$ as the number of active users in each of the other $n$ cells. The received signal at the base station receiver in the $0^{\text {th }}$ cell is given by $\operatorname{Re}\left(r(t) e^{j \omega_{c} t}\right)$ where

$$
\begin{aligned}
r(t)= & \sum_{u=1}^{K} \sqrt{P} W^{0, u}\left(t-\tau_{u}\right) A^{0}\left(t-\tau_{u}\right) e^{j \phi_{u}}+n(t) \\
& +\sum_{i=1}^{n} \sum_{l=l}^{L} \sqrt{P} \alpha_{p} W^{i, l}\left(t-\tau_{i, l}\right) A^{i}\left(t-\tau_{i, l}\right) e^{j \phi_{i, l}}
\end{aligned}
$$

$\phi_{u}=\sigma_{u}-\omega_{c} \tau_{u}, \phi_{i, l}=\sigma_{i, l}-\omega_{c} \tau_{i, l}$ and $n(t)$ is the complex white Gaussian noise process with two sided power spectral density of $N_{0} / 2$. The relative time delay random variables $\tau_{u}, \tau_{i, l}$ are mutually independent and the random variables $\phi_{u}, \phi_{i, l}$ denote the relative phase shifts of modulo $2 \pi$. The attenuation due to path loss and shadowing is denoted as $\alpha_{p}$. Without loss of generality, we consider the transmission of Walsh codes for the $\mathrm{k}$-th user in the $0^{\text {th }}$ cell and the two PN codes $\left(P_{I}(t), P_{Q}(t)\right)$ are neglected in the subsequent analysis.

For the k-th user, the relative time delay of the desired signal is equal to zero and the transmitted code is assumed the first Walsh code $W_{1}(t)$. For simplicity, perfect power control is assumed and system synchronization is slightly imperfect such that the relative time delay $\tau_{u}$ is uniformly distributed over $[0$,

$T_{W}$ ) for all $u$ and $u \neq k$. The receiver employs a bank of $M$ matched filters for coherent detection of each possible codeword. The received signal is therefore coherently detected and non-coherently combined to generate the decision statistic variable $S_{p}^{k}$ given by $s_{p}^{k}=\left(Z_{p}^{1, k}\right)^{2}+\left(Z_{p}^{2, k}\right)^{2}$. The combined output of the matched filter $Z_{p}^{1, k}$ and $Z_{p}^{2, k}$ can be written [3] as

$$
\begin{aligned}
& Z_{1}^{1, k}+j Z_{1}^{2, k}=\frac{1}{2 \sqrt{T}} \int_{0}^{T} r(t) A^{0^{*}}(t) W_{1}(t) d t \\
& =\sqrt{P T}\left\{\delta\left(W_{v}^{k}(t) W_{1}(t)\right) k^{j \theta_{k}}+\frac{1}{2 T}\left[\sum_{\substack{u=1 \\
u \neq k}}^{K} x_{u}\left(R_{v-1,1}^{u}\left(\tau_{u}\right)+\hat{R}_{\nu, 1}^{u}\left(\tau_{u}\right)\right) k^{j \phi_{u}}\right.\right. \\
& \left.\left.+\sum_{i=1 l=1}^{n} \sum_{p}^{L} \alpha_{p} x_{i, l} W^{i, l}\left(t-\tau_{i, l}\right) A^{i}\left(t-\tau_{i, l}\right) e^{j \phi_{i, l}} W_{1}(t) A^{0^{*}}(t)\right]\right\} \\
& \quad+\eta_{I}+j \eta_{Q}
\end{aligned}
$$

If $W_{p}(t)=W_{v}^{k}(t), \delta\left(W_{v}^{k}(t) W_{p}(t)\right)=1$; otherwise 0 . The complex conjugate of the $0^{\text {th }}$ cell signature sequence is denoted as $A^{0^{*}}(t)$. The notations $x_{u}, x_{i, l}$ are the voice activity random variables with probabilities of $\operatorname{Pr}\left(x_{i}=\right.$ $+1)=\alpha$ and $\operatorname{Pr}\left(x_{i}=0\right)=(1-\alpha)$ for all $u, i$ and $l$. The two zero-mean Gaussian random variables $\eta_{l}$ and $\eta_{Q}$ are of variance $N_{0} / 2$. The continuous time partial crosscorrelation functions [7] of Walsh codes are denoted as $R_{v-1, p}^{u}(\tau), \hat{R}_{v, p}^{u}(\tau)$.

\section{SYSTEM ANALYSIS}

A. Single Cell System. A single base station system having $K$ active users is considered as a single cell system. The second term of the equation (5) is known as the multiple access interference $I_{m}$ given by

$$
I_{m}=\sqrt{\frac{P}{4 T}} \sum_{\substack{u=1 \\ u \neq k}}^{K} x_{u}\left(R_{v-1,1}^{u}\left(\tau_{u}\right)+\hat{R}_{u, 1}^{u}\left(\tau_{u}\right)\right) e^{j \phi_{u}}
$$

and it can be written [7] as

$$
\begin{gathered}
I_{m}=\sqrt{\frac{P}{4 T}} \sum_{\substack{u=1 \\
u \neq k}}^{K} x_{u}\left(C_{v-1,1}^{u}(-M) \hat{R}_{\phi}(\tau)+C_{\nu-1,1}^{u}(1-M) R_{\phi}(\tau)\right. \\
\left.+C_{v, 1}^{u}(0) \hat{R}_{\phi}(\tau)+C_{v, 1}^{u}(1) R_{\phi}(\tau)\right)^{j \phi_{u}}
\end{gathered}
$$

For simplicity, assume that the first Walsh code $W_{1}(t)$ is not transmitted from any other users so that $C_{v-1,1}^{u}(-M)=0$ and $C_{v, 1}^{u}(0)=0$. The $I_{m}$ is written as

$I_{m}=\sqrt{\frac{P}{4 T}} \sum_{\substack{u=1 \\ u \neq k}}^{K} x_{u} \tau_{u}\left(a_{M-1}^{v-1} a_{0}^{1}+\sum_{j=0}^{M-2} a_{j}^{v} a_{j+1}^{1}\right) e^{j \phi_{u}}$

Assuming sequences are random binary in equation (8), the variance of $I_{m}$ is given [7] by

$\operatorname{Var}\left(I_{m}\right)=\frac{P T_{w}^{2}}{12 T}(K-1) \alpha M$

According to the Central Limit Theorem, the multiple access interference $I_{m}$ becomes a Gaussian distributed random variable. Therefore, the variance of the random variable $Z_{1}^{1, k}$ in (5) can be written as 
$\operatorname{var}\left(Z_{1}^{1, k}\right)=\sigma_{\text {tot }}^{2}=\frac{P T_{w^{\prime}}^{2}}{24 T}(K-1) \alpha M+\frac{N_{0}}{2}$

The mean is given by $E\left(Z_{1}^{1, k}\right)=\sqrt{P T}$ for $\mathrm{p}=1$ and $E\left(Z_{1}^{1, k}\right)=0$ for $\mathrm{p}=2,3, \ldots, M$. After the non-coherent combining at the receiver, it can be easily shown [6] that the probability density function of the decision statistic output variable $S_{p}^{k}$ is given by the Rician distribution for $p=1$ and Rayleigh distribution for $p=2,3, \ldots, M$. The average bit error probability $P_{e}$ can thus be written as

$P_{e}=\frac{2^{m-1}}{2^{m}-1} \sum_{a=1}^{M-1}\left(\begin{array}{c}M-1 \\ a\end{array}\right) \frac{(-1)^{a+1}}{a+1} e\left(\frac{E_{w^{a}}}{2(a+1) \sigma_{t o t}^{2}}\right)$

where symbol energy $E_{w}=m E_{b}$ as in [3].

B. Multiple Cell System: Consider a typical hexagonal cell cellular system of cell radius $R$ and user density $\rho$ as in [5]. It is reasonable to assume that all the transmitting signals from users in other cells are independent and regarded as interference to the intended base station. As the pilot PN sequences at the intended base station are different from that in the other cells, the receiving interfering signal from other cells is thus regarded as random binary code. The interference $I_{o t h}[5]$ can thus be approximated by

$I_{\text {oth }}=\sqrt{P / 4 T} \iint_{\mathrm{S}_{0}} \alpha_{p} x d A$

where $x$ is voice activity random variable. For the propagation attenuation $\alpha_{p}$, we adopted the notations and assumptions by Viterbi et al.[5] who also assumed that the interference $I_{o t h}$ is Gaussian distributed.

1. Hard Handoff Consider a power controlled multiple-cell cellular system in which the hard handoff is employed. Assuming that the mobile user density over all the cells is uniformly distributed, the average interference $E\left(I_{\text {oth }}\right)$ from users in the other cells to the $0^{\text {th }}$ cell base station [5] can be written as

$E\left(I_{o t h}\right)=\sqrt{\frac{P}{4 T}} \alpha e^{(b \beta \sigma)^{2}} \iiint_{S_{0}}\left(\frac{r_{i}}{r_{0}}\right)^{4} \rho d A$

where " $\bar{S}_{0}$ " denotes the area outside the $0^{\text {th }}$ cell area and $\beta=\ln (10) / 10$. The parameter $r_{j}$ is the distance between the mobile user and the $j^{\text {th }}$ base station. The corresponding variance is given by,

$\operatorname{Var}\left(I_{\text {oth }}\right)=\operatorname{Var}\left[\sqrt{\frac{P}{4 T}} \iiint_{\mathrm{S}_{0}}\left(\frac{r_{i}}{r_{0}}\right)^{4} x e^{b \beta\left(\varepsilon_{i}-\varepsilon_{0}\right)} \rho d A\right]$ where $\left(\varepsilon_{i}-\varepsilon_{0}\right)$ is Gaussian distributed with zero mean and variance of $2 \sigma^{2}$. Assuming the spatial whiteness [4] of blockage variable, the variance is equal to

$$
\operatorname{Var}\left(I_{o t h}\right)=\frac{P}{4 T} \alpha e^{2(b \beta \sigma)^{2}}\left(e^{2(b \beta \sigma)^{2}}-\alpha\right) \iint_{S_{0}}\left(\frac{r_{i}}{r_{0}}\right)^{8} \rho \mathrm{dA}
$$

Assuming the circular cell radius $R$ of unity for simplicity, the user density $\rho$ of value $2 K /(3 \sqrt{3})$ and $L=K$, the mean and the variance of interference from other cells are given by $E\left(I_{\text {oth }}\right)=0.401708 \sqrt{P} K$ and $\operatorname{Var}\left(I_{\text {oth }}\right)=2.536513 P K$, respectively.

2. Soft Handoff amongst the two nearest candidate base stations: Consider a similar system as in the hard handoff above. Instead of using the hard handoff algorithm, the soft handoff is employed and two cases are considered as follows. The first case is to consider the mobile user within the area " $\mathrm{S}_{0}$ " and the other case is outside the area " $\mathrm{S}_{0}$ ", as depicted in Figure 2 of [5]. A user who is within the area " $\mathrm{S}_{0}$ " and currently connected to and power controlled by the $1^{\text {st }}$ cell, the average interference [5] to the $0^{\text {th }}$ cell can be written as

$$
E\left(I_{i n}\right)=\sqrt{\frac{P}{4 T}} \alpha e^{(b \beta \sigma)^{2}} \iint_{\mathrm{S}_{0}}\left(\frac{r_{1}}{r_{0}}\right)^{4} Q\left[\sqrt{2} b \beta \sigma+\frac{M_{1}-M_{0}}{\sqrt{2} b \sigma}\right] \rho d A
$$

where $M_{j}=40 \log \left(r_{j}\right)$ and $Q(x)=\int_{x}^{\infty} e^{-\left(y^{2} / 2\right)} d y / \sqrt{2 \pi}$.

The corresponding variance is given by

$$
\operatorname{Var}\left(I_{i n}\right)=\operatorname{Var}\left\{\sqrt{\frac{P}{4 T}} \int_{\mathrm{S}_{0}} \int\left(\frac{r_{1}}{r_{0}}\right)^{4}\left[x 10^{\left(\zeta_{1}-\zeta_{0}\right) / 10} ; \mathrm{r}_{1}^{4} 10^{\zeta_{1} / 10}<\mathrm{r}_{0}^{4} 10^{\zeta_{0} / 10}\right] \mathrm{ddA}\right\}
$$

where $\zeta_{i}=a \varepsilon+b \varepsilon_{i}$ as defined in [5]. Therefore, $\left(\varepsilon_{1}-\varepsilon_{0}\right)$ is Gaussian distributed with zero mean and variance of $2 \sigma^{2}$. The variance is thus be equal to

$$
\begin{aligned}
\operatorname{Var}\left(I_{i n}\right)= & \frac{P \alpha e^{2(b \beta \sigma)^{2}}}{4 T} \iint_{\mathrm{S}_{0}}\left(\frac{r_{1}}{r_{0}}\right)^{8}\left[e^{2(b \beta \sigma)^{2}} Q\left(2 \sqrt{2} b \beta \sigma+\frac{M_{1}-M_{2}}{\sqrt{2} b \sigma}\right)\right. \\
& \left.-\alpha\left(Q\left[\sqrt{2} b \beta \sigma+\frac{M_{1}-M_{2}}{\sqrt{2} b \sigma}\right]\right)^{2}\right] \rho d A
\end{aligned}
$$

Consider the second case where a mobile user is currently connecting to the $1^{\text {st }}$ cell in the area " $\overline{\mathrm{S}}_{0}$ " outside the area " $\mathrm{S}_{0}$ ". In this case, the average interference to the $0^{\text {th }}$ cell [5] is given by,

$$
E\left(I_{\text {out }}\right)=2 \sqrt{\frac{P}{4 T}} \alpha e^{(b \beta \sigma)^{2}} \iiint_{S_{0}}\left(\frac{r_{1}}{r_{0}}\right)^{4} Q\left[\frac{b \beta \sigma}{\sqrt{2}}+\frac{M_{1}-M_{2}}{\sqrt{2} b \sigma}\right] \rho d A
$$

The corresponding variance is equal to 


$$
\begin{gathered}
\operatorname{Var}\left(I_{\text {out }}\right)=\operatorname{Var}\left\{\sqrt{\frac{P}{4 T}} \iiint_{\overline{\mathrm{S}}_{0}}\left(\frac{r_{1}}{r_{0}}\right)^{4}\left[x 10^{\left(\zeta_{1}-\zeta_{o}\right) / 10} ; \mathrm{r}_{1}^{4} 10^{\zeta_{1} / 10}<\mathrm{r}_{2}^{4} 10^{\zeta_{2} / 10}\right] d A\right\} \\
+\operatorname{Var}\left\{\sqrt{\frac{P}{4 T}} \iint \frac{r_{\mathrm{S}_{0}}}{r_{0}}\right)^{4}\left[x 10^{\left(\zeta_{1}-\zeta_{o}\right) / 10} ; \mathrm{r}_{2}^{4} 10^{\zeta_{2} / 10}<\mathrm{r}_{1}^{4} 10^{\zeta_{1} / 10} \mathrm{pdA}\right\}
\end{gathered}
$$

$=I_{1}+I_{2}$

Following the similar manipulation as for equation (18), $I_{l}$ can be written as

$$
\begin{aligned}
I_{1}= & \frac{P \alpha e^{2(b \beta \sigma)^{2}}}{4 T} \iint_{\mathrm{S}_{0}}\left(\frac{r_{1}}{r_{0}}\right)^{8}\left[e^{2(b \beta \sigma)^{2}} Q\left(\sqrt{2} b \beta \sigma+\frac{M_{1}-M_{2}}{\sqrt{2} b \sigma}\right)\right. \\
& \left.-\alpha\left(Q\left[\frac{b \beta \sigma}{\sqrt{2}}+\frac{M_{1}-M_{2}}{\sqrt{2} b \sigma}\right]\right)^{2}\right] \rho d A
\end{aligned}
$$

As $\varepsilon_{1}$ and $\varepsilon_{2}$ are independent identical random variables, it can be easily deduced that $I_{1}=I_{2}$. The variance of cell interference is thus equal to

$$
\begin{aligned}
\operatorname{Var}\left(I_{\text {out }}\right)= & \frac{P}{2 T} \alpha e^{2(b \beta \sigma)^{2}} \iiint_{\mathrm{S}_{0}}\left(\frac{r_{1}}{r_{0}}\right)^{8}\left[e^{2(b \beta \sigma)^{2}} Q\left(\sqrt{2} b \beta \sigma+\frac{M_{1}-M_{2}}{\sqrt{2} b \sigma}\right)\right. \\
& \left.-\alpha\left(Q\left[\frac{b \beta \sigma}{\sqrt{2}}+\frac{M_{1}-M_{2}}{\sqrt{2} b \sigma}\right]\right)^{2}\right] \rho d A
\end{aligned}
$$

Assuming the cell radius $R$ of unity, the user density $\rho$ of value $2 K /(3 \sqrt{3})$ and $L=K$, the mean and variance of interference from other cells in the area " $\mathrm{S}_{0}$ " are given by $E\left(I_{\text {in }}\right)=0.080136 \sqrt{P} K$ and $\operatorname{Var}\left(I_{\text {in }}\right)=0.036074 P K$, respectively. For the area " $\overline{\mathrm{S}}_{0}$ ", the mean and variance are equal to $E\left(I_{\text {out }}\right)=0.226 \sqrt{P} K$ and $\operatorname{Var}\left(I_{\text {out }}\right)=$ $0.163323 P K$, respectively. Summing the interference for the two areas, the average and the variance of the interference are given by $E\left(I_{\text {oth }}\right)=E\left(I_{\text {in }}\right)+E\left(I_{\text {out }}\right)$ and $\operatorname{Var}\left(I_{\text {oth }}\right)=\operatorname{Var}\left(I_{\text {in }}\right)+\operatorname{Var}\left(I_{\text {out }}\right)$, respectively. Therefore, the variance of the receiver output variable $Z_{l}^{1, k}$ in (5) can be written as

$$
\operatorname{Var}\left(Z_{1}^{1, k}\right)=\sigma_{\text {tot }}^{2}=\frac{P T_{w}^{2}}{24 T}(K-1) \alpha M+\frac{N_{0}}{2}+\operatorname{Var}\left(I_{\text {oth }}\right)
$$

The mean is given by $E\left(Z_{1}^{1, k}\right)=\sqrt{P T}+E\left(I_{o t h}\right)$ for $p=1$ and $E\left(Z_{1}^{1, k}\right)=E\left(I_{o t h}\right)$ for $\mathrm{p}=2,3, \ldots . M$. It can be shown [6] that the pdf of the variable $S_{p}^{k}$ become the Rician distribution function for $p=1,2,3, \ldots, M$. As a close form expression [pp261, 6] can not be further derived for the multiple cell system, numerical integration is employed to evaluate the average bit error probability instead.

\section{RESULTS}

Figures 1,2,3,4,5 and 6 depict the BER performance of the $M$-ary DS/CDMA cellular system in the presence of the additive white Gaussian channel noise for single and multiple-cells system. For $M=64$, observe that the BER performance is sensitive to a small change of $E_{b} / N_{0}$ in the region of $5-10 \mathrm{~dB}$. When $K$ is small (less than 30 , say), the error performance for the soft handoff is marginally superior to that of hard handoff. It is also less sensitive to $K$ and the superiority increases with increasing $K$. It has also shown that the error performance for multiple cells system with soft handoff is marginally degraded compared to that of the single cell system.

\section{CONCLUSION}

The BER performance for the $M$-ary DS/CDMA cellular system in the presence of the additive white Gaussian channel noise and multiple-cell interference was investigated. Assuming perfect power control and non-perfect system synchronization, both the hard handoff and soft handoff were also examined. A close form expression for the BER had been derived for a single cell CDMA system whereas numerical integration was employed for the multiple cells CDMA systems instead.

\section{REFERENCES}

[1] Kyoung Il Kim, "On the Error Probability of a DS/CDMA System with Noncoherent $M$-ary orthogonal Modulation," in Proc. Of VTC'92, pp 482-485, Denver, CO, 1992

[2] Qi Bi, "Performance Analysis of a CDMA Cellular System," in Proc. Of VTC'92, pp 43-46 Denver, CO, 1992

[3] Een Kee Hong, Ki Jun Kim and Keum Chan Whang, "Performance Evaluation of DS-CDMA System with $M$-ary Orthogonal Signaling," in IEEE Transactions on Vehicular Technology, Vol. 45, pp 57-63, Feb. 1996.

[4] K. S. Gilhousen, I. M. Jacobs, R. Padovani, A. J. Viterbi, and C. E. Wheatly, "On the Capacity of a Cellular CDMA System," in IEEE Transactions on Vehicular Technology, Vol. 40, pp 303-312, May, 1991.

[5] Andrew J. Viterbi, Audrey M. Viterbi, Klein. S Gilhousen, and Ephraim Zehavi, "Soft Handoff Extends CDMA Cell Coverage and Increase Reverse Link Capacity," IEEE Journal of Selected areas in Communications, Vol. 12, pp 1281-1288, Oct. 1994.

[6] John G. Proakis "Digital Communications" Third Edition, McGraw-Hill Book Company, 1995.

[7] James S Lehnert and Michael B. Pursley, "Error Probabilities for Binary Direct-Sequence Spread-Spectrum Multiple-Access Communications with Random Signature Sequence" in IEEE Transactions on Communications, , vol. 35, pp 87-98, Jan. 1987.

[8] TIA/EIA/IS-95 Interim Standard, Mobile station-Base Station Compatibility Standard for Dual-Mode Wide band Spread Spectrum Cellular System, Telecommunication Industry Association, July 1993 


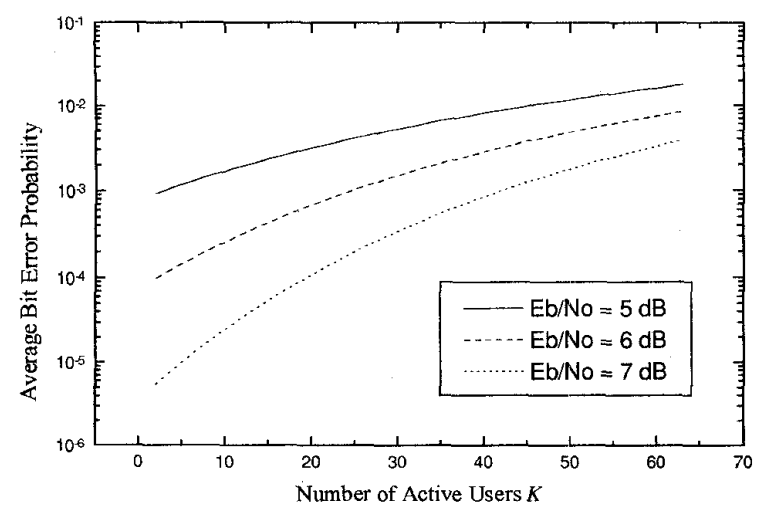

Figure 1. The error performance of the $M$-ary DS/CDMA system in a single cell system.

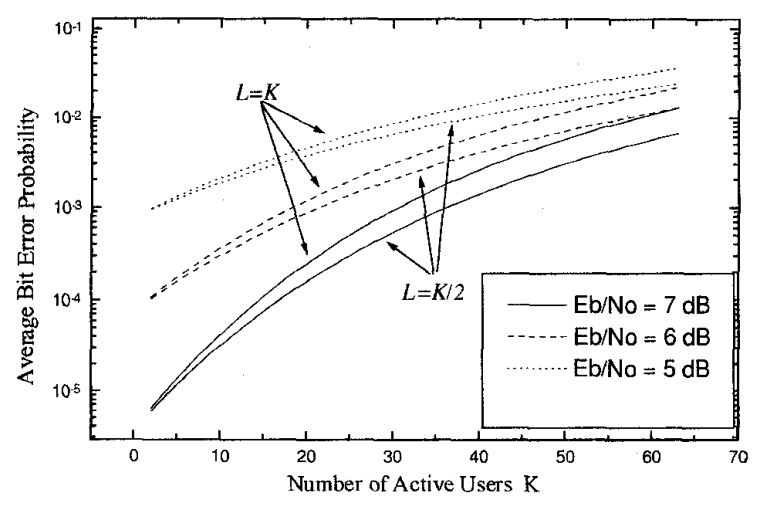

Figure 3. The error performance of the $M$-ary DS/CDMA system with hard handoff in a multiple cell cellular system.

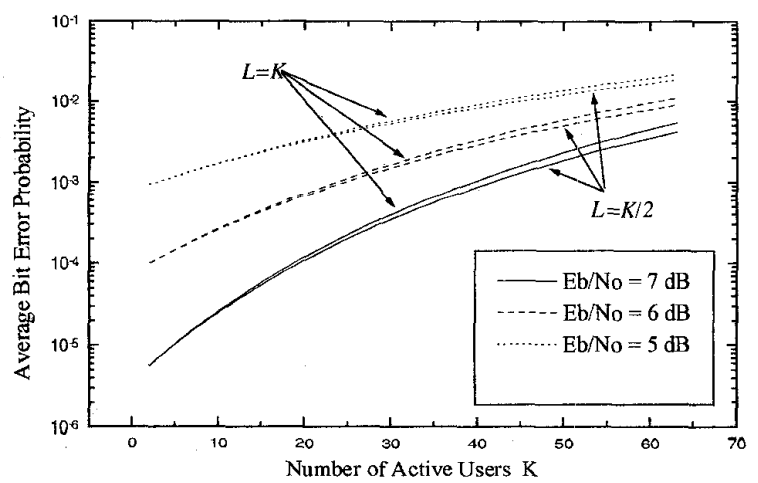

Figure 5. The error performance of the $M$-ary DS/CDMA system with soft handoff in a multiple cell cellular system.

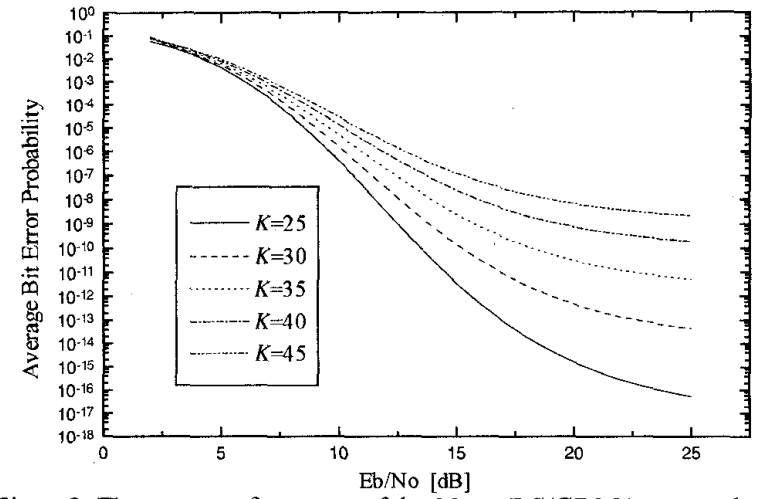

Figure2. The error performance of the $M$-ary DS/CDMA system having $K$ number of users in a single cell system.

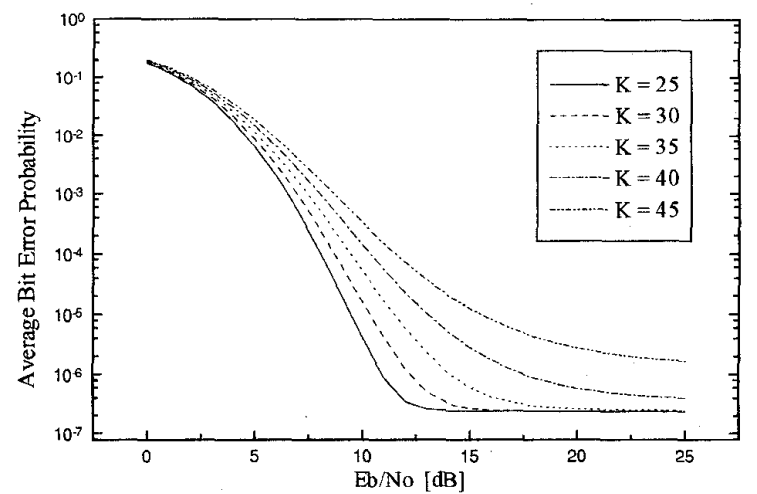

Figure 4 . The error performance of the $M$-ary DS/CDMA with hard handoff in a multiple cell cellular system.

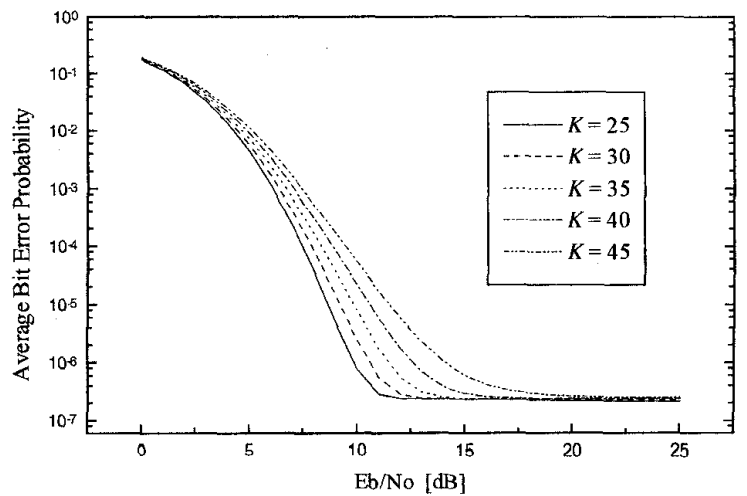

Figure 6. The error performance of the $M$-ary DS/CDMA system with soft handoff in a multiple cell cellular system. 